\title{
El Emprendimiento e innovación. Una perspectiva de desarrollo económico, social y sustentable en la Zona 6
}

\section{Entrepreneurship and innovation. A perspective of economic, social and} sustainable development in Zone 6

Edison Becerra Molina. ${ }^{1}$

\section{Recibido: 21-05-2020 /Aceptado: 20-06-2020 / Publicado: 03-07-2020}

\section{Abstract. DOI: https://doi.org/10.33262/concienciadigital.v3i3.1297}

This article is aimed at building a theoretical-academic management model for the development of innovative entrepreneurship policies and programs at the Catholic University of Cuenca, which contributes to the professional profile in the different careers, leading to offering a methodological proposal for its components. integrators, with political feasibility for its implementation at UCACUE. For this, it was necessary to apply information collection instruments to students of the eighth and ninth cycles of sixteen careers at the Catholic University of Cuenca from the period March-August 2019, which served to prepare the researcher's Doctoral Thesis called "Theoretical-academic management model for the development of innovative entrepreneurship policies and programs at the Catholic University of Cuenca", aims to enhance the capabilities of the student population and expand their opportunities in terms of job and income generation, in a way collective and sustained, based on the development and productive vocation of the enterprises in zone 6 and their economic potential, so that they can autonomously exercise their rights as citizens and improve their quality of life in a sustainable way. In order to fulfill this purpose, it was necessary to have the political commitment of institutional actors, and strategic guidelines to foster and promote an entrepreneurial culture and productive links, innovation and research. The study is located in the positivistic, empirical-analytical quantitative epistemic paradigm, corresponds to the field design, not experimental and transectional, and is located at a descriptive level. Key informants were selected through sampling. The techniques of the survey and the interview were applied, and as instruments of data collection; the questionnaire and the script of questions to the different social actors, in order to take into account, the observations and theoretical,

\footnotetext{
${ }^{1}$ Universidad Católica de Cuenca, Cuenca, Ecuador, jbecerram@ucacue.edu.ec
} 
methodological contributions of the productive, business and government sectors. The instruments were validated through expert judgment. The study is oriented in the description of the administrative and academic processes regarding the ideas, purposes and problematizations that revolve around entrepreneurship, for which it was necessary to develop the projects, programs and an integrated plan for UCACUE, which allows contributing to the development of the entrepreneurial culture in the training of leading professionals and entrepreneurs as one of the transversal axes of the University's processes, through the design of the institutional strategy for research, development, innovation and incubation, and contributing to the construction of learning processes, based on the creation of added value in curricular and extracurricular academic processes, considering University policies linked to the sustainable development of the territory. Among the conclusions presented in this first article, the majority of students consider that the important projects in their training are research, because they build knowledge from intellectual and practical links, it is appreciated that entrepreneurship projects are needed to strengthen the entrepreneurial spirit, which requires training students with responsibility, commitment, effort, dedication, perseverance and a spirit of work.

Keywords: Education, curriculum, entrepreneurship, innovation and employability.

\section{Resumen.}

El presente artículo está orientado a construir un modelo de gestión teóricoacadémico para el desarrollo de políticas y programas de emprendimiento innovador en la Universidad Católica de Cuenca, que aporte al perfil profesional en las diferentes carreras, conducente a ofrecer una propuesta metodológica por sus componentes integradores, con viabilidad política para su implementación en la UCACUE. Para ello fue necesario realizar la aplicación de instrumentos de recolección de información a estudiantes de los octavos y novenos ciclos de dieciséis carreras de la Universidad Católica de Cuenca del periodo marzo- agosto del 2019, que sirvió para la elaboración de la Tesis Doctoral del investigador denominada "Modelo de gestión teórico- académico para el desarrollo de políticas y programas de emprendimiento innovador en la universidad católica de cuenca", tiene como objetivo potenciar las capacidades de la población estudiantil y ampliar sus oportunidades en materia de generación de empleo e ingresos, de manera colectiva y sostenida, basándose en el desarrollo y la vocación productiva de los emprendimientos en la zona 6 y su potencial económico, para que puedan ejercer con autonomía sus derechos como ciudadanos/as y mejorar su calidad de vida de manera sostenible. para cumplir con este propósito fue necesario contar con el compromiso político de los actores institucionales, y las directrices estratégicas para fomentar y promover la cultura emprendedora y la vinculación productiva, la innovación y la investigación. El estudio se ubica en el paradigma 
epistémico positivista, cuantitativo empírico-analítico, se corresponde con el diseño de campo, no experimental y transeccional, y se ubica en un nivel descriptivo. Mediante un muestreo se seleccionó a los informantes claves. Se aplicó las técnicas de la encuesta y la entrevista, y como instrumentos de recolección de datos; el cuestionario y el guion de preguntas a los diferentes actores sociales, con el objeto de tomar en cuenta las observaciones y aportes teóricos, metodológicos de los sectores productivos, empresariales y de gobierno. Los instrumentos fueron validados a través de juicio de expertos. El estudio se orienta en la descripción de los procesos administrativos y académicos en cuanto a las ideas, propósitos y problematizaciones que giran alrededor del emprendimiento, para lo cual fue necesario desarrollar los proyectos, programas y un plan integrado para la UCACUE, que permita contribuir al desarrollo de la cultura emprendedora en la formación de profesionales líderes y emprendedores como uno de los ejes transversales de los procesos de la Universidad, mediante el diseño de la estrategia institucional de investigación, desarrollo innovación e incubación y aportar a la construcción de procesos de aprendizaje, basado en la creación de valor agregado en los procesos académicos curriculares y extracurriculares, considerando las políticas Universitarias vinculadas al desarrollo sostenible del territorio. Entre las conclusiones que se presentan en este primer artículo se tiene que mayoritariamente los estudiantes consideran que los proyectos importantes en su formación son los de investigación, por que construyen conocimiento a partir de vínculos intelectuales y prácticos, se aprecia que hace falta proyectos de emprendimiento para fortalecer el espíritu emprendedor, que exige formar alumnos con responsabilidad, compromiso esfuerzo, dedicación perseverancia y espíritu de trabajo.

Palabras clave: Educación, currículum, emprendimiento, innovación y empleabilidad.

\section{Introducción.}

Los efectos de la pandemia por la propagación del COVID-19 están sacudiendo las estructuras socioeconómicas a nivel local, regional, y nacional. Los impactos se estiman en cifras astronómicas de pérdidas de vidas y empleos, el gobierno y el sector productivo, están desplegando medidas sanitarias y económicas para frenar el avance de la epidemia con gran incertidumbre aún sobre la duración del periodo de emergencia sanitaria y las consecuencias reales de la pandemia sobre la población y el tejido productivo.

Por lo tanto, se hace necesario promover políticas de Emprendimiento e Innovación, con el objetivo de instituir sinergias mediante el trabajo articulado, armónico y coordinado de los actores del ecosistema de emprendimiento e innovación. Por ello con un trabajo conjunto y alineado entre la academia, los sectores productivos y el gobierno, permitirá la reactivación económica de la región y del país. 
El presente trabajo de investigación se encuentra centrado en el eje transversal de la cultura emprendedora, la política de emprendimiento innovador, los elementos curriculares y las competencias, necesarias para generar un modelo de gestión teóricoacadémico para el desarrollo de políticas y programas de emprendimiento innovador en la Universidad Católica de Cuenca-UCACUE, que aporte a la formación de nuevos emprendedores en las diferentes carreras en correspondencia con la demanda económicosocial del territorio de la zona 6.

El estudio partió de la descripción de los procesos administrativos y académicos en cuanto a las ideas, propósitos y problematizaciones que giran alrededor del currículo, bajo el cual se desarrollan actividades, proyectos y programas curriculares y extracurriculares para la UCACUE, que permitieron constituir, establecer, comprobar y programar los objetivos, contenidos, curriculares y extracurriculares a ser implementados en el proceso enseñanza-aprendizaje, que estén articulados con la cultura emprendedora y su vinculación con el estudio, las bases conceptuales, así como los enfoques teóricos que sirvieron de orientación durante todo el proceso investigativo.

El proceso de sistematización metodológica que sirvió para delimitar las características del contexto, hacia dónde y a quiénes se orienta el estudio, de allí que la investigación se ubica en el paradigma epistémico positivista, con enfoque cuantitativo empíricoanalítico, el tipo de indagación al que se recurrió en función de los objetivos propuestos, está íntimamente ligado con el diseño de campo y nivel descriptivo, por cuanto el investigador analizó las técnicas de emprendimiento innovador en la Universidad Católica de Cuenca, y su relación con los sectores productivos y gubernamentales, para ello se utilizaron la técnica de la encuesta siendo el instrumento el cuestionario que fueron dirigidos a estudiantes de los octavos y novenos ciclos, tomando en cuenta las observaciones y aportes teóricos, metodológicos, productivos, empresariales y de políticas públicas.

\section{Metodología.}

Esta investigación se enmarcó en el ámbito del emprendimiento, ya que se tuvo conciencia que existió una realidad que descubrir, construir e interpretar, contempla su propia metodología, por cuanto comúnmente se aplica únicamente al mundo de los negocios. la ruta metodológica parte del paradigma de investigación positivista, tipo de investigación diseño de campo no experimental y transeccional o transversal con apoyo documental, nivel de investigación fue la investigación descriptiva, Arias (2016), señala que: "La investigación descriptiva consiste en la caracterización de un hecho, fenómeno, individuo o grupo, con el fin de establecer su estructura o comportamiento" (p.24). De allí que se corresponde con el nivel descriptivo en función del objetivo general planteado, la población estuvo estructurada por 4 sectores escogidos, corresponde en esta oportunidad referirnos al universo de la población estudiantil de los octavos y novenos ciclos de las carreras de las Unidades Académicas, considerando la población, para el año 2019 se tuvo 4.260 estudiantes, mediante muestreo estratificado se determinó la muestra 
$\mathrm{n}=246$, la validez de contenido fue realizado mediante el juicio de tres (3) expertos con experiencia en el área de emprendimiento y metodología.

Por otra parte, es necesario destacar que mediante la técnica de la encuesta, se elaboró el instrumento del cuestionario, para validar la "Construcción del modelo de gestión teóricoacadémico", el mismo tomó en cuenta los criterios de factibilidad curricular (cinco dimensiones), de precisión (5 dimensiones), de utilidad (4 dimensiones), de ética (3 dimensiones), de pertinencia (4 dimensiones), y contenido (4 dimensiones), cada uno de ellos con un conjunto de dimensiones en estricta vinculación con los objetivos de investigación, con opciones de respuesta dicotómicas y observaciones abiertas que a juicio de los validadores fuesen importante para mejorar la construcción definitiva del modelo.

En el diseño y validación del Modelo de Gestión Teórico- Académico para el Desarrollo de Políticas y Programas de Emprendimiento Innovador en la Universidad Católica de Cuenca, fue validado, considerando desde el punto de vista didáctico, los indicadores que tomaron en cuenta los validadores expertos, representados por la Academia, sector Gubernamental y los Sectores Productivos. Los indicadores considerados en la validación fueron la factibilidad curricular, precisión, utilidad, ética, pertinencia y contenidos. Por su parte, fue importante analizar en primer lugar, la pertinencia del coeficiente de consistencia interna de Cronbach, el misma habla, sobre el grado en que los ítems miden la homogeneidad.

Para determinar la confiabilidad del cuestionario elaborado, se procedió aplicar una prueba piloto a 30 estudiantes de los octavos y novenos ciclos, que no forman parte de la muestra y que corresponden a la Sede Azogues de la Universidad Católica de Cuenca, y a la provincia del Cañar, todos ellos con características similares a los sujetos de este estudio, administrando una escala, de 18 ítems, a una muestra de $n=30$. De acuerdo con los resultados anteriores, se concluyó que la escala tiene una confiabilidad de consistencia interna muy alta, suficientemente alta como para ser considerada fiable. En conclusión, la confiabilidad del instrumento fue de 0,9 muy alta, así mismo, fue importante la utilización de la herramienta estadística para el desarrollo de la investigación, recurriéndose a la estadística descriptiva, lo que permitió la recolección, organización y presentación de datos, facilitando, con el apoyo de cuadros, tablas y figuras, los resultados obtenidos de la aplicación de los cuestionarios en relación con los objetivos planteados del presente estudio, mediante el software libre SPSS, versión 26.

Con respecto, al procesamiento y análisis de los datos obtenidos a lo largo del proceso de investigación, se realizó, considerando el uso de la triangulación, validez, confiabilidad y contrastación de los instrumentos.

\section{Fundamentación Teórica.}

El marco teórico referencial comprende aspectos teóricos, conceptuales, legales, situacionales de la realidad que forman parte de la presente investigación, garantizando 
de esta manera la integración e interrelación de la teoría con la metodología y objetivos de investigación.

Es oportuno indicar que la educación empresarial empieza a tener un papel fundamental en los campos de estudios Universitarios, puesto que la estadística del informe del Banco Mundial (2014), sostiene que se crean nuevas empresas cada vez con mayor celeridad. No obstante, la quiebra y cierre de nuevas empresas también es alta. De allí la trascendencia de una buena educación empresarial, puesto que varios autores como Drucker, Schumpeter, Stevenson, Aronsson, Arieu, entre otros, contribuyen con sus estudios al éxito de los empresarios si han recibido una formación relacionada.

Druker (1985), señala que el emprendimiento es una práctica, es un comportamiento, es decir una conducta y una actitud, por eso, y retomando la idea de lo anteriormente mencionado, se expresa por medio de la educación y se modifican comportamientos, puede entonces señalarse, que existe una estrecha relación entre espíritu emprendedor y educación.

El espíritu emprendedor exhibe una doble apariencia, es así que por un lado encontramos el espíritu emprendedor que supone saber lanzar nuevos ideales con independencia, capacidad de tomar riesgo, con seriedad, con visión, con aforo de autoridad y con capacidad de solucionar los problemas organizacionales, así como los que se le presentan a las personas en su proceso de interacción social. Por consiguiente, educar el espíritu emprendedor exige formar a los alumnos en la responsabilidad, el compromiso, el esfuerzo, la dedicación, la perseverancia y el espíritu de trabajo. Cabe destacar, que el rol del capital humano y la experiencia del emprendedor, es determinante para el desempeño a largo plazo en el emprendimiento tecnológico. Wright et. al., (2007).

La declaración de Mar del Plata (2015), postula que "la formación emprendedora e innovadora debe introducirse en el currículo de la educación formal en todos los niveles desde educación básica a educación superior e iniciarse a edad temprana". Por su parte, la Comisión Económica para América Latina -CEPAL (2018), fomentó estados abiertos, participativos e innovadores para construir un nuevo vínculo con los ciudadanos, estos deben estar concebidos para anticiparse al cambio y adaptarse a un contexto global rápidamente cambiante y cada vez más complejo.

Según, la Agenda 2035 para la educación superior en el Ecuador, los fines de la educación superior se plasman en su Constitución: Art. 350.- El sistema de educación superior tiene como finalidad la formación académica y profesional con visión científica y humanista; la investigación científica y tecnológica; la innovación, promoción, desarrollo y difusión de los saberes y las culturas; la construcción de soluciones para los problemas del país, en relación con los objetivos del régimen de desarrollo (Asamblea Constituyente, 2008, p.162).

Según, la investigadora ecuatoriana, Lasio (2016), afirma que la educación en emprendimiento y la creación de negocios incide favorablemente en la intención de 
emprender, especialmente de aquellos emprendedores que se capacitaron durante sus estudios primarios y secundarios, y que el hecho de estar expuesto a algún tipo de educación en emprendimiento influye en la percepción de poseer conocimientos y habilidades necesarias para crear un negocio. Pero no se da este tipo de formación con la amplitud y profundidad necesaria en las Universidades del Ecuador en la actualidad.

Con respecto al nivel académico, conocimiento y competencias, según Grandi y Grimaldi (2005), sostienen que el valor de aspectos tales como las habilidades son importantes para articular la información, el conocimiento y las competencias. De acuerdo con el curriculum y emprendimiento como una aproximación teórica, debo indicar que la conceptualización del currículo se origina en el latín y la palabra tiene relación con "Carrera"; dando a entender un nivel determinado de aprendizaje en el que lo aprendido, las experiencias, entre otros. De cada una de las personas es su propio currículo. Las asignaturas según los niveles, sus contenidos, en relación con los fines educativos nacionales es un currículo a nivel global macro curricular, en esa orientación, Torres (2016) en su libro: Diseño curricular por competencias, muestra que el currículum es una serie estructurada de resultados buscados en el aprendizaje. Para Lawton (1973), es el engranaje de todos los aspectos de la situación de enseñanza y aprendizaje.

En torno a las tecnologías de la información y la comunicación, conocidas como TIC, son el conjunto de tecnologías desarrolladas para gestionar información y enviarla de un lugar a otro. Incluyen un abanico de soluciones muy amplio. Incluyen las tecnologías para almacenar información y recuperarla después, enviar y recibir información de un sitio a otro, o procesar información para poder calcular resultados y elaborar informes", (Lee y Jones, 2008).

Las TIC son herramientas teórico conceptuales, soportes y canales que procesan, almacenan, sintetizan, recuperan y presentan información de la forma más variada. Los soportes han evolucionado en el transcurso del tiempo (telégrafo óptico, teléfono fijo, celulares, televisión) ahora en ésta era podemos hablar de la computadora y de la Internet. El uso de las TIC representa una variación notable en la sociedad y a la larga un cambio en la educación, en las relaciones interpersonales y en la forma de difundir y generar conocimientos, Ciberespacio profesional, (2011). La innovación tecnológica se acostumbra a asociar con la idea de progreso y búsqueda de nuevos métodos, partiendo de los conocimientos que le anteceden, a fin de mejorar algo que ya existe, dar solución a un problema o facilitar una actividad, Rodríguez (2011).

La innovación es una acción continua a lo largo del tiempo y abarca diferentes campos del desarrollo humano, a su vez el campo de la tecnología se caracteriza por un continuo avance. La innovación, por lo tanto, es una de las características de la tecnología que supone la creación de nuevos dispositivos, en muchos casos, a partir de la modificación de elementos ya existentes.

La innovación conlleva a la competitividad y desarrollo de bienes y servicios tecnológicos de alta calidad. De allí que, que la innovación es la solución de problemas, en esta época 
de incertidumbre, es habitual escuchar a políticos hablar de la innovación como la fórmula para salir de la crisis. Sin embargo, se podría argumentar que el sector financiero, en el que se originó la crisis actual, ha sido uno de los más innovadores en los últimos años, incluso, podría decirse que fueron esas innovaciones financieras las que la provocaron. Pero no sólo eso: casi una década antes, la evolución de Internet y todo el sector de las tecnologías de la información y las comunicaciones (TIC) también generó una burbuja bursátil que acabó estallando. La innovación se está desplazando a los países emergentes. Durante las últimas décadas han sido receptores de tecnología y conocimiento, y en el proceso han aprendido.

Por su parte, Ley Orgánica de Emprendimiento e Innovación, (2020), en el Artículo 2.Objetivos de la ley, sostiene que entre los objetivos de la ley es fortalecer la interacción y sinergia entre el sistema educativo y actores públicos, privados, de economía mixta, popular y solidaria, cooperativista, asociativa, comunitaria y artesanal del sistema productivo nacional.

\section{Resultados.}

En este capítulo se procede al análisis e interpretación de los resultados, según Palella y Martins (2012), la interpretación de los resultados consiste en inferir conclusiones sobre los datos codificados, basándose en las operaciones intelectuales de razonamiento lógico e imaginación, ubicando tales datos en el contexto teórico.

La interpretación de los resultados estadísticos fue un elemento crucial para la formulación del modelo. La tabulación e interpretación de los cuestionarios dirigidos a estudiantes de los octavos y novenos ciclos de 16 carreras de la Universidad Católica de Cuenca, son un total de 18 ítems, para el presente análisis se han seleccionado únicamente cinco, los más relevantes.

Figura 1. En la carrera académica se forma en emprendimiento.

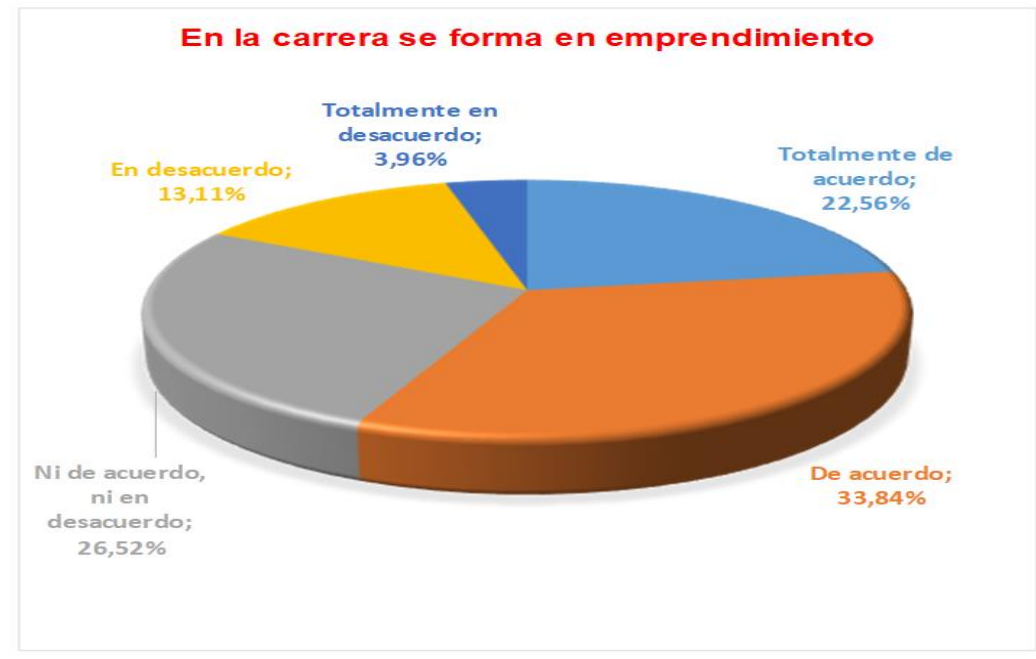

Fuente: Elaboración propia. 
La información recogida permitió determinar que un alto porcentaje de estudiantes encuestados, representado por un $22.56 \%$ está totalmente de acuerdo en que la carrera forma en emprendimiento, 33,84\% manifiestan estar de acuerdo, al unir ambas tendencias representan el 56,40\%, quienes señalan que, en las aulas universitarias de la Universidad Católica de Cuenca, se da formación en emprendimiento. Sin embargo, es pertinente tomar en cuenta un porcentaje significativo de encuestados $(26,52 \%)$ quienes expresan que están ni de acuerdo ni en desacuerdo, mientras que en la categoría en desacuerdo se ubica un $13,11 \%$ y totalmente en desacuerdo un $3.96 \%$, al unir estas tres tendencias se tiene un porcentaje total del $43,59 \%$, esto es indicativo que es necesario revisar la formación emprendedora que actualmente, se desarrolló en la UCACUE con la finalidad de promover un modelo de cultura emprendedora para formar ciudadanos económicamente activos, con la finalidad de aumentar su capital humano.

¿En su formación qué opciones usted considera importantes para el desarrollo de sus competencias?

Figura 2. Desarrollo de competencias

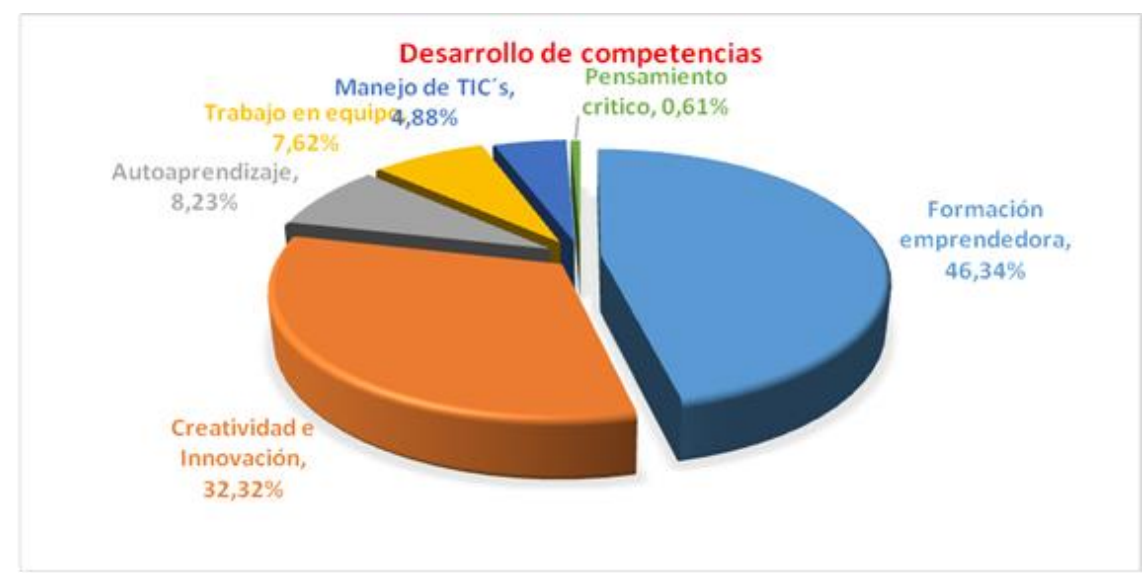

Fuente: Elaboración propia (2020).

Los estudiantes encuestados manifiestan en su mayoría, que el emprendimiento es importante para el desarrollo de competencias en su formación, según la Figura 2, representa el 46,34\%, por tales razones el emprendimiento se ha convertido en los últimos años en una estrategia social de formación para el desarrollo de competencias, que puede permitir a los estudiantes asumir una cultura emprendedora, realizar proyectos empresariales para el autoempleo con un mayor grado de certeza y posibilidad de mejorar la calidad de vida.

Con respecto a la creatividad e innovación, el 32,32\% sostiene que la creatividad y la innovación es el activo principal de futuro de las empresas y organizaciones, mientras que el $8,23 \%$, considera que el autoaprendizaje, es importante para desarrollar competencias, igualmente el 7,62\%, trabajo en equipo, el $4.88 \%$, manejo de Tic y finalmente el 0,61\%, pensamiento crítico. Del análisis de datos llama la atención, que los estudiantes respondieron con tendencia porcentual mínima en cuanto al manejo de las 
TIC y el pensamiento crítico, competencias fundamentales para desarrollarse en el marco de acción de una cultura emprendedora, por lo demás, elementos sustantivos que deben ser tomados en cuenta en el modelo teórico académico que se desarrolló en el presente estudio, en correspondencia con los objetivos de investigación.

¿Cuál de los siguientes proyectos le parece importante en su formación?

Figura 3. Proyectos en la formación

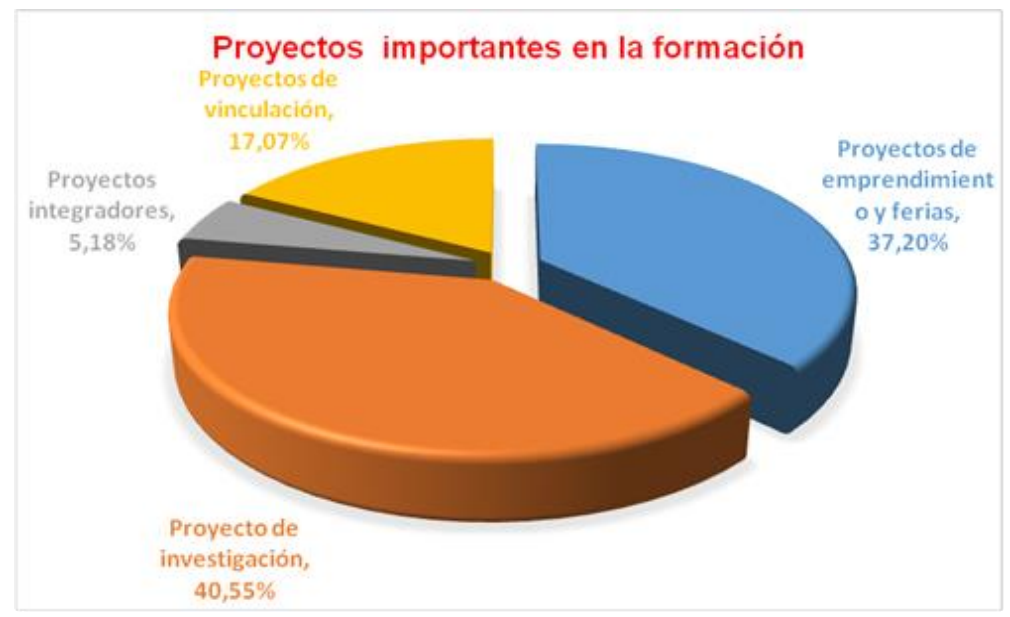

Fuente: Elaboración propia.

La información recogida permitió determinar que los proyectos importantes en la formación son en un 40,55\%, los proyectos de investigación, por lo tanto, la Universidad utiliza los recursos a su alcance para fortalecer la calidad de la formación de sus programas dando a sus estudiantes diversas dimensiones de experiencias y conocimientos relevantes, en particular una experiencia de investigación que sea genuina y significativa, la necesidad por encontrar explicaciones de lo que sucede a su alrededor, de crear e Innovar, cabe destacar que los programas de formación académica y las áreas de investigación, ayudan a los estudiantes a construir su conocimiento a partir de vínculos intelectuales y prácticas de la investigación, así mismo los proyectos de emprendimientos y ferias tienen una aceptación conforme la Figura 8 del 37,20\%, existen proyectos de emprendimiento, ferias y otras actividades extracurriculares, que los estudiantes venían realizando hasta el año 2018, en la actualidad a raíz de la jubilación de la persona responsable, llama la atención, no se realiza actividad alguna, el 17.07\% sostienen que vienen realizado proyectos de vinculación, que han dado lugar muchos de ellos, a impulsar el emprendimiento y la importancia de componentes que inciden en la sostenibilidad, la generación de riqueza, empleo y la dinamización de la economía, lo que termina en beneficio de la sociedad, por último con 5.18\% proyectos integradores, al respecto estudiantes y docentes de las diferentes Unidades Académicas de la Universidad Católica de Cuenca en cada ciclo, ponen de manifiesto y muestran los proyectos integradores de saberes, muchos de ellos se deberían articular al emprendimiento, de esta forma deberían promover e incentivar el desarrollo del comercio y la empleabilidad a través de la creatividad e innovaciones tecnológicas. 
¿Cuáles de las siguientes estrategias para desarrollar cultura emprendedora, según su criterio, fomentan los profesores en su carrera?

Figura 4. Estrategias para desarrollar cultura emprendedora

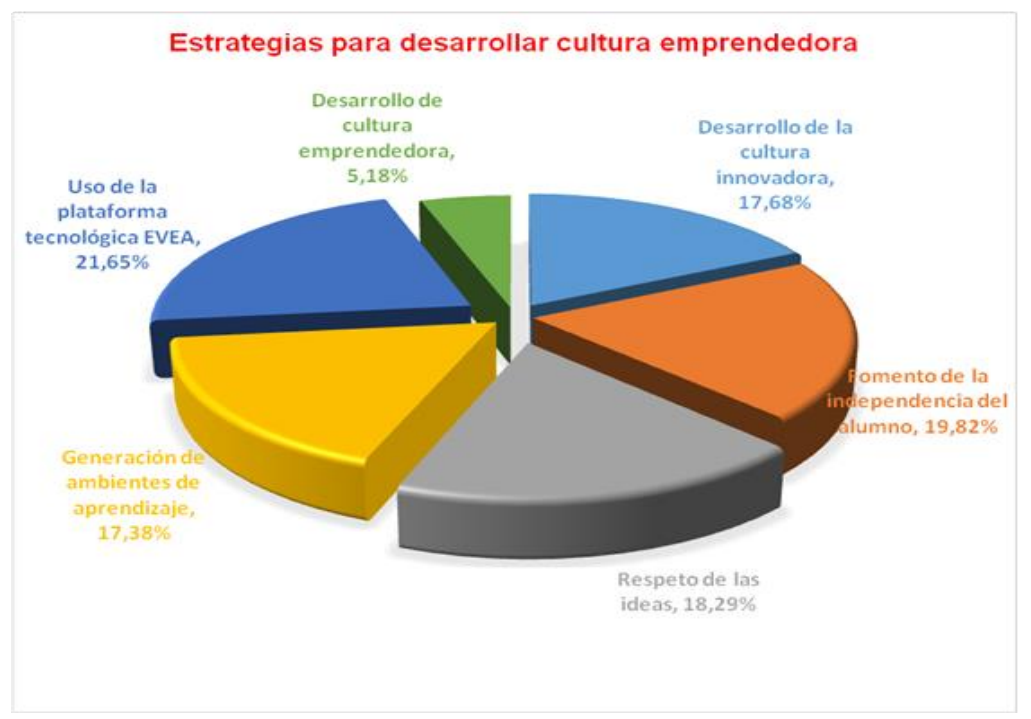

Fuente: Elaboración propia.

En torno al indicador del desarrollo de la cultura que fomentan los profesores en la carrera, manifestaron que es la utilización de la plataforma tecnológica EVEA, en un $21,65 \%$, manifiesta su acuerdo. En torno a este aspecto, la misma es una plataforma web que brinda respaldo digital a medios de divulgación o cursos o aulas virtuales, que permite interactuar con los estudiantes, además dispone de recursos diversos como aplicaciones informáticas, lecciones, foros, chat, talleres, tareas, wiki, videos, archivos, demás cuestionarios y actividades para fomentar el intercambio y la interacción, entre el profesor y el estudiante, para ello la UCACUE, cuenta con el entorno virtual de enseñanza aprendizaje (EVEA), Por otro lado indicaron que el fomento de la independencia del alumno, con el $19.82 \%$, aquí estaría la independencia cognoscitiva, la misma radica en la capacidad del estudiante para manifestar y resolver los problemas epistemológicos, con sus propias ímpetus, el respeto de las ideas con el $18.29 \%$, son propósitos y problematizaciones que giran alrededor del currículo, bajo el cual se desarrollarán proyectos, programas y planes de estudios en la UCACUE, que permite constituir, establecer, comprobar y programar los objetivos, contenidos, competencias básicas, programación didáctica, material, métodos y metodologías a ser implementados en el proceso enseñanza-aprendizaje.

El Desarrollo de la cultura innovadora con el 17.68\% de los estudiantes encuestados, de allí nace la creación de empresas innovadoras y de base tecnológica, siendo uno de los motores del crecimiento económico de cualquier región desarrollada. Pero para que estas empresas existan, deben preexistir emprendedores con una idea de negocio de la que posteriormente surgirán estas empresas. La generación de ambientes de aprendizaje con el $17.38 \%$, tiene como premisa garantizar una educación inclusiva, equitativa y de 
calidad y promover oportunidades de aprendizaje, en cuanto al desarrollo de la cultura emprendedora que fomentan los profesores, esta es la de menor aceptación por los estudiantes encuestados, con el 5,18\%, esto preocupa, toda vez que los programas y proyectos de cultura emprendedora asocian la voluntad de diferentes ámbitos de gestión de la administración como es el caso de la educación y empleo, empresa y Juventud, al tiempo que es necesario diseñar acciones de manera transversal, explicitando la visión que la Universidad tiene acerca del emprendimiento y su desarrollo en la sociedad.

¿Qué tipo de vinculación conoce usted con respecto al modelo triple hélice de la relación Universidad-Empresa-Gobierno?

Figura 5. Vinculación modelo triple hélice de la relación Universidad Empresa Gobierno

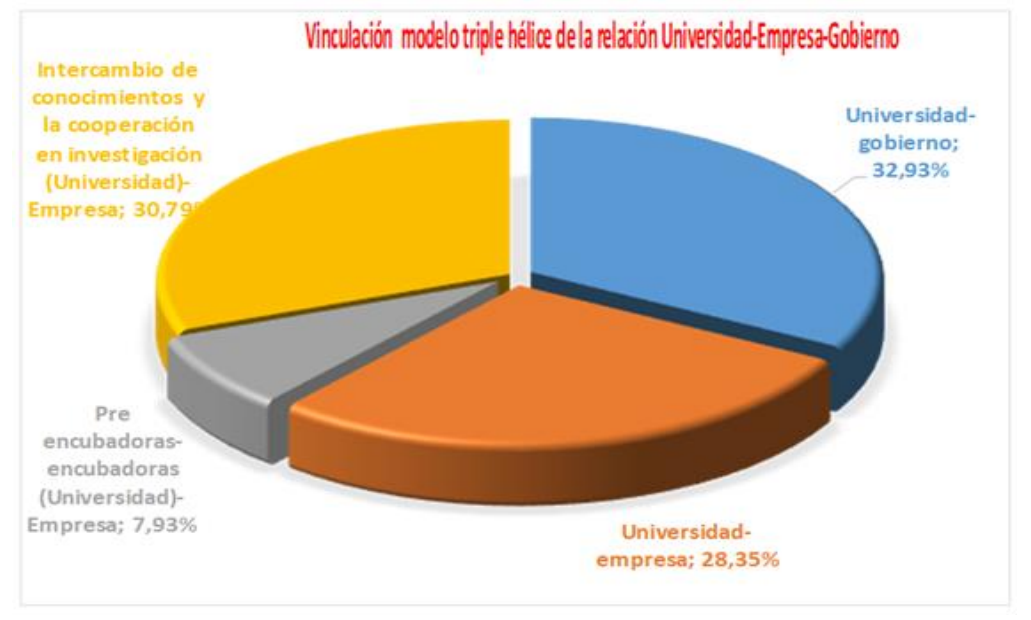

Fuente: Elaboración propia.

Al analizar la Figura 5, con respecto al modelo triple hélice de la relación UniversidadEmpresa-Gobierno, los estudiantes encuestados sostuvieron que en un 32,93\% conocen la Vinculación Universidad- Gobierno, en este sentido consideraron que está vinculada al eje 2: Economía al Servicio de la Sociedad, por cuanto a este eje se toma como punto de partida el hecho de que la economía debe estar al servicio de la sociedad. Es así que nuestro sistema económico del Ecuador, por definición constitucional, es una economía social y solidaria. Para tal fin está conformado por los objetivos 4, 5, y 6 del PNDTV, enfocándose al sistema económico social y solidario, es decir, al servicio de la población que permita garantizar los derechos. Una vez analizado los objetivos, es de resaltar que el objetivo $\mathrm{N}^{\mathrm{o}}$ 5. Promueve e impulsa la productividad y competitividad para el crecimiento económico sostenible, de manera redistributiva y solidaria, para tal fin el, país aún debe alcanzar las condiciones que anhela para una economía social y solidaria al servicio de la sociedad y que garantice el ejercicio de los derechos.

El 30,79\% consideraron que el intercambio de conocimientos y la cooperación en investigación (Universidad)-Empresa de allí que para la Universidad, el ser humano no 
es un factor más de la producción, debe tener supremacía sobre el capital, por tales razones, es fundamental erradicar la pobreza y extrema pobreza de manera sostenible, a través de la generación de trabajo de calidad, con remuneraciones dignas y seguridad social, lo cual implica desarrollar una economía que reduzca las asimetrías del mercado laboral, incorporando mayor conocimiento y capacidad de investigación e innovación, y que cuente con una política industrial clara. Parte de la innovación está en introducir nuevos paradigmas de transformación productiva como los que se encuentran alrededor de la economía circular e industrial.

Con relación a la vinculación Universidad-Empresa, el 28,35\% de los estudiantes encuestados sostuvieron que sería muy importante que tanto el gobierno como las empresas deberían promover una política industrial para generar una economía más competitiva a través de la incorporación del conocimiento; y, por ende, de especialistas que con sus innovaciones generen valor y riqueza. Para que esto se dé, se requieren fuertes incentivos económicos que sin duda redituarán en beneficios.

De igual forma, el 7,93\% Pre incubadoras- incubadoras (Universidad)-Empresa, los estudiantes encuestados manifestaron que la Universidad Católica de Cuenca, en su filosofía institucional debe proyectar ser una Universidad formadora de profesionales líderes y emprendedores para acompañar a los estudiantes y docentes a los procesos de creación y fortalecimiento de empresas innovadoras y para ello debe trabajar en la concreción del Reglamento del Centro de Incubación de Empresas Estudiantiles y Desarrollo Empresarial e impulsar para comenzar conformando un equipo consultivo y gestores de Incubación, en las áreas: Marketing Mix, Producción, Economía, Contabilidad y Finanzas.

Se contrastaron y triangularon las encuestas administradas incluso a los diferentes actores de la UCACUE y ejecutivos empresariales, posteriormente de manera cuantitativa en base a los ítems se procedió a realizar las comparaciones correspondientes, demostrando todas tendencia similar, que sirvió de base para la construcción de la propuesta del modelo.

\section{Propuesta.}

Se ha considerado necesario dentro del presente trabajo investigativo la implementación de un modelo de gestión teórico-académico para el desarrollo de políticas y programas de emprendimiento innovador en la Universidad Católica de Cuenca UCACUE, que aporte a la formación de nuevos emprendedores en las diferentes carreras en correspondencia con la demanda económico- social del territorio de la zona 6.

Como es lógico, la formación y desarrollo de la cultura emprendedora de jóvenes Universitarios, con sentido crítico, promotores de la participación, creativos en innovadores con competencias de análisis económico de entornos financieros, con la finalidad de crear con ideas alguna actividad nueva, y/o líneas de negocios generadoras de empleo, tomando en cuenta las necesidades más acuciantes a nivel local, regional y nacional. 
Es conveniente destacar, que se conoce como emprendimiento a la cualidad y capacidad que tiene una persona para preparar un pensamiento de hacer algo nuevo e innovador y/o un proyecto empresarial, mediante ideas y oportunidades. El emprendimiento es un vocablo inmensamente utilizado en el ambiente empresarial, en virtud de su relacionamiento con la creación de alguna actividad innovadora, empresas, nuevos productos o innovación de los ya existentes.

En modelo de gestión teórico - académico para el desarrollo de políticas y programas de emprendimiento innovador en la UCACUE, que se propone contiene los procesos del modelo gestión, partiendo desde las directrices estratégicas del modelo de emprendimiento, difundiendo la cultura emprendedora, fomentando la creación de empresas encaminadas hacia la innovación, promoviendo la cooperación a través de la integración productiva, conduciendo la investigación y propiciando el desarrollo hacia la innovación, proponiendo los programas con sus respectivos proyectos de emprendimiento a nivel interno y externo a ser implementados en el periodo 2020 - 2024, estructurado como respuesta a los desafíos planteados en el Plan Estratégico Institucional de la UCACUE.

En coherencia a lo anteriormente descrito en el grafico $\mathrm{N}^{\circ} 6$, se propone a corto plazo promover programas y proyectos referentes a concursos, premios y otros incentivos dirigidos a motivar y facilitar la cultura emprendedora y consecuente creación de empresas, tanto a corto y mediano. Líneas de actuación que debería promover la UCACUE, y que estaría dada mediante programas de capacitación continua de capacitación de emprendimiento innovador, aquí se propone realizar el proyecto 1 y 2 , estarían dados seminarios y talleres sobre fomento a la cultura emprendedora; y los emprendedores como agentes del desarrollo local respectivamente.

Por otra parte, un programa de eventos académicos estructurados de emprendimiento, aquí estarían los proyectos 3, 4 y 5, así el proyecto plaza que incluye producción y ventas de la UCACUE, luego el proyecto del salón del emprendimiento innovador y por último las visitas empresariales. En cuanto al programa del Club de Emprendedores están los proyectos 6, 7, 8 y 9, allí se encuentran: el intercambio de experiencias emprendedoras, el concurso de oratoria y libro leído en el ámbito de la cultura emprendedora, la capital semilla para los emprendedores de la UCACUE; y, por último, la difusión académica del propósito de la formación emprendedora.

Así mismo, se estructura un programa del sistema a los graduados, con los proyectos 10 , 11 y 12; que incluye directorio de los graduados emprendedores, Una experiencia exitosa de la UCACUE, y el proyecto aprendamos a ser emprendedores. Con respecto al programa de difusión de la cultura emprendedora en la UCACUE, está el proyecto 13, este incluye Una revista "Soy emprendedor de la UCACUE". De igual forma, debe impulsar la UCAUCE, un programa de pre incubación de empresas, aquí se considera el proyecto 14, introducción a la cultura emprendedora y ecosistema de emprendimiento e innovación. La UCACUE debe desarrollar el programa de incubación de empresas, aquí 
se sugiere situar el proyecto 15, la incubación de empresas del Centro de Incubación de Empresas Estudiantiles y Desarrollo Empresarial- CIDEM.

Por último, el programa de servicio de apoyo empresarial a la colectividad, en el que estaría el proyecto 16, con las redes de apoyo al emprendimiento productivo e incubación, llamado también clínica de Mipymes, La situación antes planteada se detalla de la siguiente manera:

Figura 6. Fomento de Programas y Proyectos encaminadas hacia la Innovación.

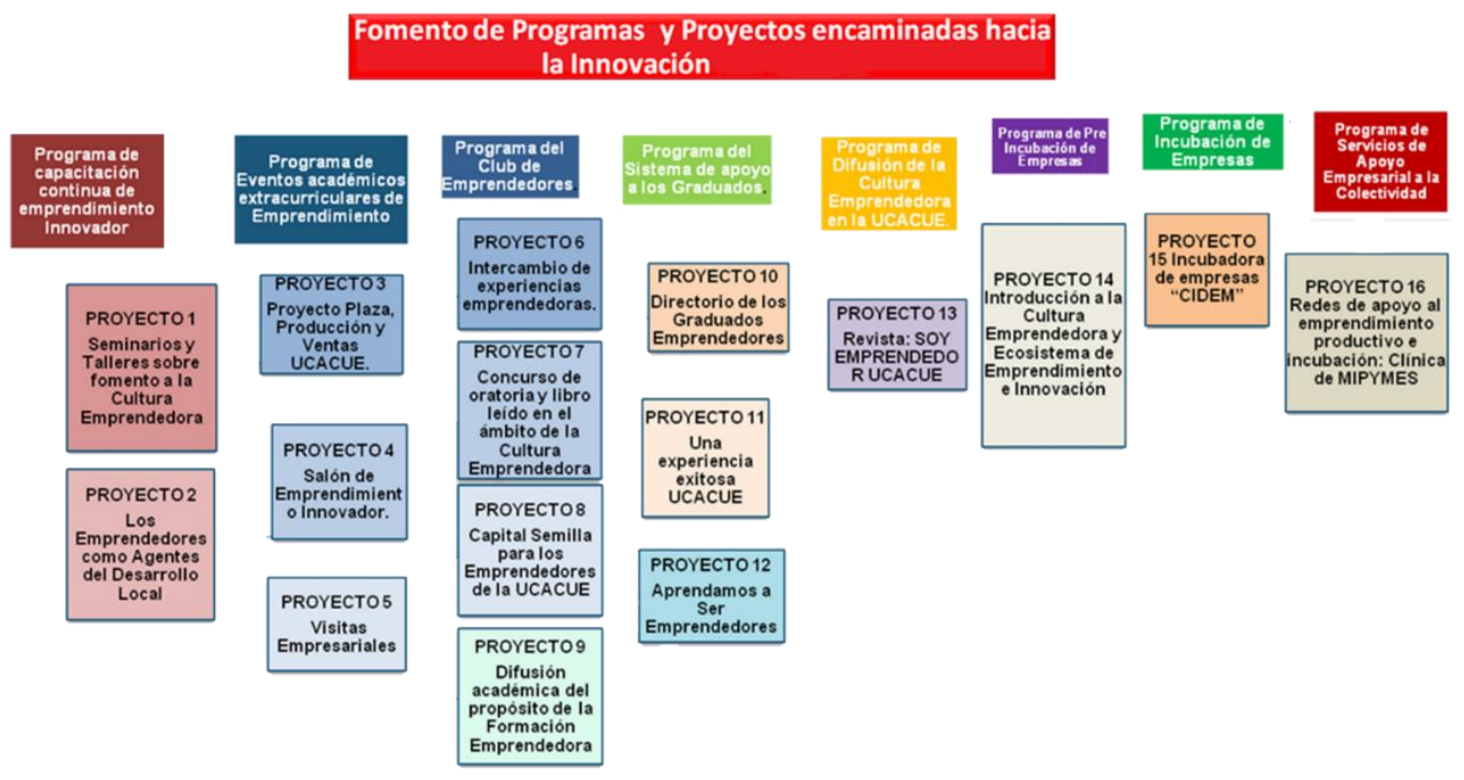

Fuente: Elaboración propia.

Es conveniente destacar que los proyectos propuestos para que lleven adelante las autoridades de la UCACUE, permitirá que los emprendimientos en la región 6, incida en la generación de riqueza, empleo y la dinamización de la economía, incidiendo en beneficio a la sociedad.

Por su parte el modelo también incluye políticas de incubación, y graduación de empresas, evaluación de las ideas de negocio, la creación de un comité se seguimiento y evaluación, indicadores y criterios de evaluación, articulados a la actual ley orgánica de emprendimiento e innovación, que tiene como objetivo establecer el marco normativo que incentive y fomente el emprendimiento, la innovación y el desarrollo tecnológico, promoviendo la cultura emprendedora e implementando nuevas modalidades societarias y de financiamiento para fortalecer el ecosistema emprendedor.

El objetivo de la propuesta es fortalecer las competencias, habilidades y destrezas para mejorar la calidad de vida de la población estudiantil, con el involucramiento de actores emprendedores que tienen la capacidad de transformación de los recursos locales para la solución de los problemas básicos de manera especial el empleo digno con valor agregado, para salir de crisis económica por la que atravesamos debido a la pandemia del 
Corona virus. El modelo permitirá definir las estructuras de apoyo, estrategias y políticas que se debe implementar para transformar a la UCACUE en una Universidad emprendedora a corto, mediano y lago largo plazo.

\section{Conclusiones:}

A continuación, se exponen las principales conclusiones, integrando los resultados de las encuestas, y la técnica de la observación:

- Con respecto a la existencia de proyectos de emprendimiento en la UCACUE, las autoridades entrevistadas, indican que actualmente no existe ningún proyecto de emprendimiento, pero que la Universidad ha implementado recintos llamados: Laboratorio de Fabricación Digital (FABLAB), que proporciona a sus usuarios el acceso a medios modernos para impulsar la creatividad; la conforman varias máquinas controladas por ordenadores, y permitirá crear ecosistemas de aprendizaje de emprendimientos innovadores, que permiten, fortalecer, organizar y dirigir el potencial del conocimiento de los actores locales, además del el Centro de Investigación, Innovación y Transferencia de Tecnología (CIITT). Que contiene siete nuevos laboratorios de biotecnología, microbiología, contaminación ambiental y aguas residuales, principios activos y seguridad alimentaria, neurociencias, simulación de tiempo real, la UCACUE ha puesto disposición de la colectividad científica local y regional.

- En relación, a la concreción de proyectos de emprendimiento, la Universidad Católica de Cuenca, de igual forma conforme el pronunciamiento de los estudiantes de los octavos y novenos ciclos en un $39,02 \%$, no existen proyectos, por tanto se tiene que impulsar y desarrollar los ocho programas y los dieciséis proyectos, con sus distintas actividades curriculares y extracurriculares, para fomentar la cultura emprendedora en los estudiantes de las diferentes carreras de la UCACUE, con calidad y calidez, mediante la cátedra de emprendimiento, que aporte a la formación de profesionales líderes y emprendedores, y contribuya de manera sostenida a la sociedad, especialmente en el territorio.

- Los resultados evidenciaron que sumado las opciones muy satisfactorio, satisfactorio y medianamente satisfactorio, los estudiantes de los octavos y novenos ciclos el 83,23\%, además de las entrevistas semiestructuradas efectuadas a las autoridades Universitarias, principales, rector, vicerrectores, Académico y de Investigación e Innovación, pusieron de manifiesto la importancia académica de la cultura emprendedora, para dar respuestas asertivas a la Región 6, como un verdadero cambio a la educación transformacional del conocimiento y el aprendizaje, que se encamina a la creación de una formación Universitaria con generación de valor agregado a través del emprendimiento innovador.

- En cuanto a las estrategias para desarrollar la cultura emprendedora, los actores estudiantes en un $20,68 \%$, que tanto el desarrollo de la capacidad innovadora, 
como el fomento de la independencia del alumno, el respeto de las ideas, la generación de ambientes de aprendizaje, el uso de la plataforma tecnológica, entorno enseñanza aprendizaje (EVEA); y, el desarrollo de cultura emprendedora, son transcendentales y coinciden que todos los enunciados son necesarias para el desarrollo de la cultura emprendedora.

- Los gerentes o empresarios, aunque no tengan una amplia experiencia emprendedora, pueden ser excelentes mentores, por consiguiente, para alcanzar la sostenibilidad y sustentabilidad de una empresa se requiere de conocimiento y experiencia en administración y finanzas.

- Del análisis de contraste de información, los estudiantes en el 40,55\%; determinaron que los proyectos importantes de formación, son los proyectos de investigación, de allí que es importante promover la investigación en el campo del emprendimiento, en coordinación con el Vicerrectorado de Investigación de la Universidad, en función de las líneas y sublíneas de investigación analizados en el presente trabajo de investigación.

- De acuerdo a los resultados alcanzados, con respecto a la formación de los profesores para el desarrollo de sus competencias, los encuestados manifestaron, estudiantes en el 96,97\%, que la búsqueda de oportunidades e iniciativas, fijación de metas, exigencia de calidad y eficiencia, e independencia y autoconfianza, sumado a creatividad e innovación, el autoaprendizaje, el trabajo en equipo y el manejo de Tics, todas estas habilidades para fortalecer las competencias son reveladoras e importantes por qué permiten elevar la calidad de la transferencia de conocimientos, de manera eficaz, mediante modernos procesos de enseñanzaaprendizaje que corresponda a las futuras demandas de la sociedad, e incitar a los estudiantes a perder el miedo de fallar y no desistir jamás de intentar una y otra vez una idea de negocio. Esta es la esencia misma del espíritu emprendedor y exactamente eso queremos inculcar a los docentes para que adquieran competencias emprendedoras, que permitan crear y desarrollar sus habilidades, y mejorar su perfil como académicos y se conviertan en profesores formadores de estudiantes, que fortalezcan la motivación y formación para la generación de emprendimientos innovadores.

- Las políticas de emprendimiento innovador en modelo de gestión académico de la Universidad Católica de Cuenca, se articulan armónicamente con los elementos sustantivos como la docencia, investigación y vinculación con la sociedad. Es por ello que se concibe como una institución que trabaja en procesos investigativos y de proyección hacia el logro de la innovación, el emprendimiento y la integración. De no haber innovación e integración, no resulta posible alcanzar la responsabilidad social que debe caracterizar a la Universidad, por ello la importancia de la ejecución de proyectos de emprendimiento así se manifiestan los estudiantes encuestados. 
- Es necesario articular las políticas y reglamentos con la cátedra de emprendimiento, por ello las autoridades sostienen que existen políticas y que actualmente se están adecuando al nuevo estatuto, una vez definido es importante que la Universidad impulse proyectos de innovación y emprendimiento, que es la ruta a seguir para crear una actitud en las estudiantes, una cultura y una capacidad en la generación de ideas de negocios y empresas y una característica del entorno competitivo.

- En el modelo de gestión para apoyar la cultura emprendedora innovadora en la UCACUE, de acuerdo a las encuestas en un promedio del $41 \%$ y entrevistas debe incluirse la cátedra de emprendimiento cuyo objetivo de esta materia es conocer y aplicar diversas estrategias para estimular la creatividad y la competitividad, e incentivando el espíritu emprendedor que le permitan poner en práctica de manera innovadora los conocimientos adquiridos en el programa tomando como base un problema estratégico o una idea nueva u oportunidad de negocio.

- Los proyectos curriculares y extracurriculares, referente a seminarios y talleres sobre fomento a la cultura emprendedora, proyecto plaza que incluye producción y ventas de la UCACUE, el proyecto del salón del emprendimiento innovador, las visitas empresariales, el club de emprendedores, el intercambio de experiencias emprendedoras, el concurso de oratoria y libro leído en el ámbito de la cultura emprendedora, el capital semilla para los emprendedores de la UCACUE; el proyecto de pre incubación de empresas, el desarrollo incubación de empresas a la que se ha denominado en el presente estudio investigativo Centro de Incubación de Empresas Estudiantiles y Desarrollo Empresarial- CIDEM, las redes de apoyo al emprendimiento productivo e incubación, a la que le he llamado Clínica de Mipymes, deben ser consideradas y ejecutados por la Universidad en el corto, mediano y largo plazo, para fortalecer la educación emprendedora y generar una mentalidad emprendedora, con habilidades y competencias, basado en la creación de valor agregado en los procesos académicos curriculares y extracurriculares de la política Universitaria, integrada de manera estructural y vinculada al desarrollo para alcanzar el bien común.

- En torno, a los criterio a considerar en un modelo de desarrollo y fortalecer la Cultura Emprendedora en la UCACUE, debe ser considerado como eje transversal en la formación profesional, por ello está declarado en los ejes sustantivos del nuevo estatuto de la Universidad, es necesario que se incorpore a otros programas de carácter institucional, con el propósito de contribuir al desarrollo del Espíritu Emprendedor en los estudiantes de la Universidad y coadyuvar al desarrollo sostenible del territorio, para mejorar la calidad de vida de la población.

- Se recomienda a las Autoridades de la Universidad Católica de Cuenca la ejecución del modelo de gestión teórico-académico para el desarrollo de políticas y programas de emprendimiento innovador, realizado por el investigador, una vez 
que se defina la política institucional, por el cambio de Estatuto Orgánico. El mismo no debe ser visto como la solución a todos los problemas en materia de emprendimiento e innovación, problemas que se pueden presentar a la hora de implementar un modelo educativo, como todo proceso nuevo, debe estar sujeto a una constante revisión y mejora de los distintos elementos involucrados en los procesos académicos de formación emprendedora con componente investigativo, que impulse la creación de ideas de negocios o empresas innovadoras y el aporte al desarrollo sostenido de la zona 6.

\section{Referencias bibliográficas:}

Agenda 2035 para la educación superior en el Ecuador (2017), Núcleo de Estudios Estratégicos sobre Democracia, Desenvolvimiento y Sustentabilidad. Quito: Ecuador.

Arias, F. (2016). El Proyecto de Investigación: Introducción a la metodología científica. ( $7^{a}$ Edición), Caracas - Venezuela. Editorial El pasillo, C.A.

Asamblea Constituyente. (2008). Constitución de la República del Ecuador.

Montecristi: Asamblea Constituyente, Gaceta oficial No 449 del 20 de octubre del 2008.

Banco Mundial (2014), América latina en movimiento, recuperado de: https://www.alainet.org/es/articulo/173175.

Ciberespacio profesional, (2011). Plataforma académica de investigación, recuperado de: http://aprendeenlinea.udea.edu. co/lms/investigacion/mod/page/view.php?id=3118

Drucker, P. (1985). La Innovación y el empresario innovador. Ed. Edhasa Página. 25-26; $35-44$.

Grandi A. y Grimaldi R. (2005). Academics' organizational characteristics and the generation of successful business ideas. Journal of Business Venturing, 20 ( 6), 821-845.

Hurtado de Barrera, J. (2010). Guía para la comprensión holística de la ciencia, Tercera Edición, Fundación Sypal: Caracas-Venzuela.

La Comisión Económica para América Latina - CEPAL (2018), Perspectivas económicas de América Latina 2018: Repensando las instituciones para el desarrollo, Edición. OCDE, Paris.

Lasio, V. (2016). Global Entrepreneurship Monitor- Ecuador, Espae - Espol, ISSN: 13903047, https://goo.gl/ApfrLS, 1-82.

Lawton, D. (1973). Cambio social, teoría educativa y plan de estudios. Londres: University of London Press. Enlace, Frances.

Lee R. y Jones O. (2008). Networks, Communication and Learning during Business Start-up: The Creation of Cognitive Social Capital. International Small Business Journal, 26 (5), 559-594.

Ley Orgánica de Emprendimiento e Innovación, (2020), Esta Ley fue publicada en el Suplemento del Registro Oficial N 151 , del 28 de febrero del 2020. 
Mar del Plata (2015). IX Workshop de Formación de Emprendedores de la Red Emprende SUR, realizado en la ciudad de Mar del Plata de Argentina los días 9, 10 y 11 de septiembre de 2015.

Naciones Unidas, Asamblea General (2012). "Iniciativa empresarial para el desarrollo" realizada el 21 de diciembre de 2012, pág. 67/202.

Rodríguez, M. (2011). La teoría del aprendizaje significativo: una revisión aplicable a la escuela actual. Revista Electrònica d'Investigació i Innovació Educativa i Socioeducativa, Santa Cruz de Tenerife, 3(1), 29-50.

Santa Palella y Feliberto Martins (2012). Metodología de la Investigación Cuantitativa, 3ra edición, FEDUPEL. Caracas-Venezuela.

SENPLADES. (2017). Plan Nacional para el Buen Vivir 2017-2021. Quito: SENPLADES, Gaceta oficial No 681 del 13 de julio del 2017.

Torres, F. (2016). Diseño Curricular por competencias, Manual para formación capacitación y aseguramiento de gestión, segunda edición:Ecuador -Ambato. Editorial Offset Ambato

Wright M., Lockett A.,Claryse B. y Binks M.(2007).University spin-out companies and venture capital. Research Policy, 35, 481-501. 
PARA CITAR EL ARTÍCULO INDEXADO.

Becerra Molina, E. (2020). El Emprendimiento e innovación. Una perspectiva de desarrollo económico, social y sustentable en la Zona 6. ConcienciaDigital, 3(3), 165185. https://doi.org/10.33262/concienciadigital.v3i3.1297

\section{\Ciencia}

El artículo que se publica es de exclusiva responsabilidad de los autores y no necesariamente reflejan el pensamiento de la Revista Conciencia Digital.

El artículo queda en propiedad de la revista y, por tanto, su publicación parcial y/o total en otro medio tiene que ser autorizado por el director de la Revista Conciencia Digital.
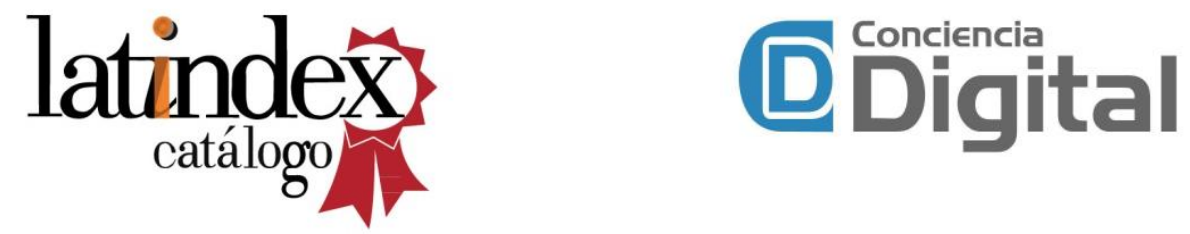